\title{
Photoresponsive Azobenzene Photonic Crystals
}

\author{
Marta Kamenjicki, ${ }^{\dagger}$ Igor K. Lednev ${ }^{\ddagger}$ and Sanford A. Asher $* \dagger$ \\ Department of Chemistry, University of Pittsburgh, Pittsburgh, Pennsylvania 15260, and \\ Department of Chemistry, University of Albany, SUNY, Albany, New York 12222
}

Received: June 9, 2004; In Final Form: July 15, 2004

\begin{abstract}
We demonstrate azobenzene photochemically driven diffraction switching of a photonic crystal consisting of a crystalline colloidal array (CCA) polymerized within a hydrogel matrix. A novel azobenzene derivative that has a large ground-state activation barrier between the cis and trans forms in water is used. The system is actuated by excitation with UV light (wavelength of $365 \mathrm{~nm}$ ), which photoisomerizes the azobenzene trans state to the cis ground state. The increased dipole moment of the cis state increases the free energy of mixing, causing a hydrogel swelling, which red-shifts the embedded CCA diffraction. Excitation with visible light photoisomerizes the cis state to the trans state, which resets the diffraction. This material acts as a memory storage material. Information is recorded and erased by exciting the photonic crystal in the UV or visible spectral region. The written information is read out completely and nondestructively by the wavelength of the Bragg diffraction (in this case, in the red).
\end{abstract}

Many groups are developing fabrication methods to produce photonic crystals with band gaps in the visible, infrared (IR), and microwave spectral regions. ${ }^{1-6}$ Photonic crystals are materials with periodic variations in their optical dielectric constants. The resulting periodic variations in the refractive index lead to the diffraction of light and to the occurrence of photonic band gaps. Light with frequencies within the band gaps cannot propagate within the photonic crystal materials. If the refractive index is purely real, then all of the light is diffracted. Photonic crystal materials provide the opportunity to control the flow of light. Photonic crystals may be the key elements of all-optical integrated circuits. ${ }^{7}$

The earliest chemical approach to fabricating photonic crystals was through the self-assembly of highly charged monodisperse colloidal particles into crystalline colloidal arrays (CCAs). ${ }^{8}$ These CCAs are complex liquids that self-assemble because of long-range electrostatic repulsions between particles into fluid, plastic, face-centered cubic (fcc) crystalline arrays. The CCAs diffract light (Bragg diffraction) in the ultraviolet (UV), visible, or near-IR range, depending on the colloidal particle array spacings. 8,9

Robust semisolid polymerized crystalline colloidal array (PCCA) materials were fabricated by imbedding the CCA into a hydrogel network (see Figure 1). ${ }^{9}$ PCCAs can be fabricated from stimuli-responsive polymer networks, ${ }^{10,11}$ where appropriate physical or chemical stimuli alter the PCCA volume, which alters the resulting CCA plane spacings and diffraction wavelengths. ${ }^{12-14}$ Previously, we described photochemically controlled PCCA photonic crystals, which operated in organic solvents such as dimethylsulfoxide (DMSO), which utilized azobenzene and spirobenzopyran photochemistry. ${ }^{15}$ In our azobenzene-functionalized PCCA, the photochemistry involved photoisomerization between the trans and cis isomers in an organic medium. In the spiropyran-modified PCCA, the pho-

* Author to whom correspondence should be addressed. Telephone: (412) 624-8570. Fax: (412) 624-0588. E-mail address: asher@pitt.edu.

$\doteqdot$ University of Pittsburgh.

$\doteqdot$ University of Albany, SUNY.
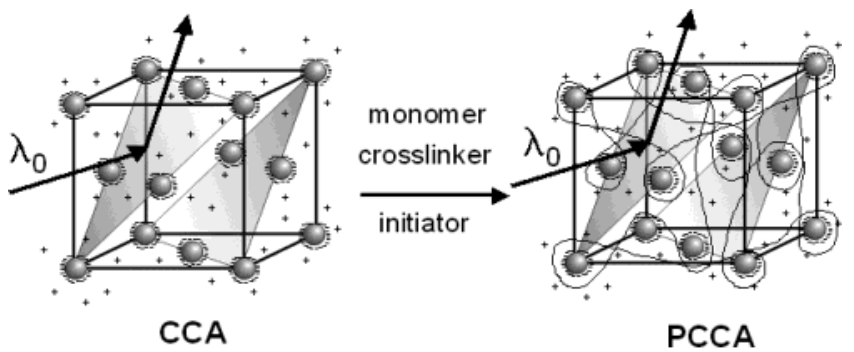

Figure 1. Polymerized crystalline colloidal array (PCCA) formed by polymerizing a hydrogel network around a crystalline colloidal array (CCA). Light is shown diffracting from the face-centered cubic (fcc) (111) planes.

tochromic behavior is due to an interconversion between the colorless closed spiro form and the colored open merocyanine form. In both systems, the formation of the more-polar form (cis-azobenzene or merocyanine) results in PCCA swelling and a red-shift in diffraction.

In this work, we demonstrate a photochemically actuated photonic crystal, where photoisomerization of a covalently attached, novel water-soluble azobenzene derivative actuates a change in the hydrogel's free energy of mixing with water. This azobenzene derivative is unusual, because it is the first, to our knowledge, to have a large activation energy barrier in the ground state between the trans and cis isomers. Although azobenzene cis derivatives are known to be stabilized for extended times in organic solvents, this is the first derivative to have a cis form that is stable in water for long periods. In this work, we have developed a CCA prepared from fluorinated colloidal particles that do not absorb near-UV light, which allows us, for the first time, to monitor the trans-azobenzene 350-nm absorption band. We find that the UV spectral changes of the trans form are completely consistent with our photoresponse mechanism.

We fabricated our photochemically controlled photonic crystal by attaching an epoxide functionality to the PCCA by copolymerizing glycidyl methacrylate, ${ }^{16-20}$ polyacrylamide, and $N, N^{\prime}$ methylenebisacrylamide around a CCA of 140-nm fluorinated 


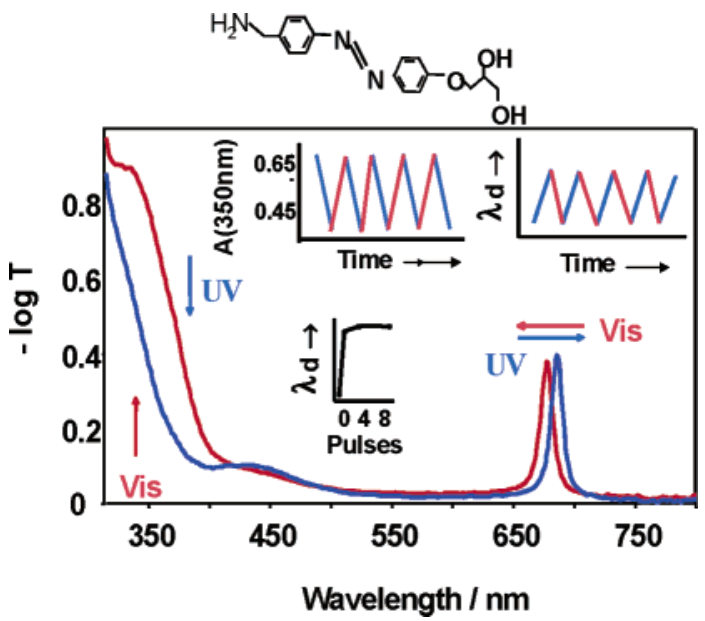

Figure 2. (Top) Structure of water-soluble azobenzene derivative. Bottom portion of figure shows the photoresponse of the azobenzene functionalized PCCA upon UV and visible light illumination. The PCCA is $\sim 80 u \mathrm{~m}$ thick and contained 5\% cross links. It was fabricated from 140-nm-diameter fluorinated colloids. The spectra show fully reversible changes in the azobenzene absorption $(300-550 \mathrm{~nm})$. The $\sim 675$-nm diffraction peak shifts $\sim 15 \mathrm{~nm}$ when subjected to alternating excitation with UV and visible light.

polymer colloidal particles. These highly charged, sulfonated, monodisperse fluorinated colloidal particles were prepared by emulsion polymerization. ${ }^{21}$ After the PCCA was fabricated, pendant epoxy groups were reacted with the primary amine of the novel water-soluble azobenzene derivative shown in Figure 2 . We determined the concentration of the azobenzene derivative that was covalently attached to the PCCA to be $\sim 5 \mathrm{mM}$.

As shown in Figure 2, this $\sim 80-\mu$ m-thick PCCA diffracts slightly more than $50 \%$ of the light in a band centered at $\sim 670$ $\mathrm{nm}$. The light is incident normal to the fcc (111) planes and is only partially diffracted, because of the small difference in refractive index between the particles $\left(n_{\mathrm{p}} \approx 1.38\right)$ and the mainly aqueous hydrogel medium $\left(n_{\mathrm{w}} \approx 1.33\right) .{ }^{21}$ PCCA made from higher-refractive-index polymer colloidal spheres, such as polystyrene $(n \approx 1.60)$, diffract essentially all of the incident light. ${ }^{12-14}$

As shown in Figure 2, excitation by UV light bleaches the 365-nm absorption band of the trans isomer and red-shifts the 670 -nm diffraction peak by $\sim 15 \mathrm{~nm}$. This red-shift results from the larger dipole moment ${ }^{22}$ of the cis-azobenzene derivative, resulting in a more-favorable mixing with the water medium. This, in turn, increases the hydrogel volume, which results in a red-shift in diffraction..$^{15}$ The change in dipole moment between the trans and cis isomers of the azobenzene is $\sim 3 \mathrm{D}^{22}$ The photochemical conversion from the trans form to the cis form is very efficient. Using azobenzene in isooctane as a reference, we have determined that the trans-to-cis quantum yield is $\Phi \approx$ 0.2 for our water-soluble azobenzene derivative in isooctane (355 $\mathrm{nm}$ excitation).

As shown in the inset in Figure 2, the diffraction can be redshifted by UV excitation (mercury lamp, $\sim 13 \mathrm{~mW} / \mathrm{cm}^{2}$ ) and blue-shifted by visible excitation (tungsten lamp, $\sim 3 \mathrm{~mW} / \mathrm{cm}^{2}$ ) within the $\sim 440-\mathrm{nm}$ cis $\pi \rightarrow \pi^{*}$ transition. The diffraction can be toggled back and forth indefinitely. Furthermore, the diffraction shift can be red-shifted continuously by moderateintensity illumination over extended time intervals or quickly switched completely by a single 3-ns 355-nm YAG laser pulse $\left(1.2 \mathrm{~mJ} / \mathrm{cm}^{2}\right)$. The time required for the red-shifted diffraction, in response to a 3-ns pulse, was shown to be $\sim 10 \mathrm{~s}$ and was limited by the macroscopic collective water and polymer diffusion time. ${ }^{15}$

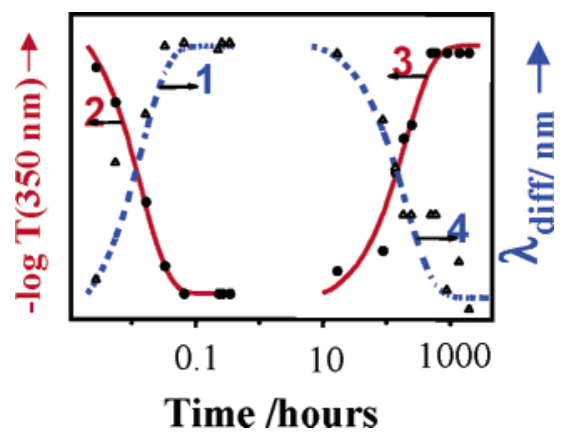

Figure 3. Time dependence of 350-nm absorbance and the diffraction wavelength with moderate-intensity incident illumination. For lines 1 and 2 , the photoisomerization is limited by the photon flux; lines 3 and 4 indicate the time course of changes that occur in the dark associated by the thermal relaxation of the cis form to the trans form over the ground-state barrier.

Extended time photoisomerization of the azobenzene derivative attached to the PCCA is shown in Figure 3. In its dark, relaxed state, the azobenzene is in its trans form and absorbs strongly at $350 \mathrm{~nm}$. The $365-\mathrm{nm}$ excitation occurs within the strong $\sim 350$-nm trans-azobenzene $\pi \rightarrow \pi^{*}$ transition, which photoisomerizes the trans-azobenzene to the cis form. Thus, the 350-nm trans-azobenzene $\pi \rightarrow \pi^{*}$ absorption bleaches the absorption band and the $\sim 440-\mathrm{nm}$ cis-azobenzene $\mathrm{n} \rightarrow \pi^{*}$ transition appears. ${ }^{23-27}$ This process can be reversed either by excitation within this $\sim 440$-nm absorption band or by the dark cis $\rightarrow$ trans conversion, which restores the $350-\mathrm{nm}$ trans absorption band and the original diffraction in $\sim 10$ days (see Figure 3). This slow thermal relaxation is very similar to the thermal isomerization of the azobenzene derivative dissolved in water.

The response time for the red-shift in diffraction is controlled either by the intensity of the incident light or by the dynamics of the restoring equilibrium balance between the free energy of mixing of the polymer hydrogel with water and the elastic restoring force of the hydrogel cross-links. ${ }^{12-15}$ For moderateintensity illumination, the response time is limited by the 350-nm photon flux, as shown in Figure 3. The higher dipole moment of the cis-azobenzene form results in an increased hydrogel polymer system in water, which decreases the free energy of mixing of the hydrogel, swelling the polymer network and causing the PCCA red-shift in diffraction. As expected, the magnitude of the red-shift in diffraction peak linearly increases as the cis-azobenzene concentration increases, as photochemistry proceeds to completion. This mechanism is clearly demonstrated by the obvious parallel kinetics of the trans absorption decrease at $350 \mathrm{~nm}$ and the red-shift in diffraction.

This PCCA functions as a novel recordable and erasable memory device. Light absorption actuates the red-shift in diffraction, which is read out in the red at wavelengths that are not absorbed and cannot induce photochemistry. This information is erased by exciting the photonic crystal with visible light. The pixel area can be smaller than $\sim 10 \mu \mathrm{m}^{2}$, an area which can easily result in a narrow diffraction band. If the diffraction shift were increased, this material could act as a display device. The response of the material is rather slow, because it is limited by the collective diffusion time of the hydrogel polymer and the required flow of water into and out of the hydrogel. However, the reading of this device is limited only by the speed with which the material can be scanned by a laser beam. This is the first example of the direct photochemical control of the wavelength of photonic crystal diffraction in water. 
Acknowledgment. This work was supported by the Office of Naval Research (Grant No. N00014-94-1-0592) and DARPA (Grant No. DAAG55-98-1-0025). We gratefully acknowledge Viktor Meier for synthesizing the water-soluble azobenzene derivative.

\section{References and Notes}

(1) Ozin, G. A.; Yang, S. M. Adv. Funct. Mater. 2001, 11, 95.

(2) Jiang, P.; Ostojic, G. N.; Narat, R.; Mittleman, D.; Colvin, V. L. Adv. Mater. 2001, 13, 389 .

(3) Goldenberg, J. M.; Wagner, J.; Stumpe, J.; Paulke, B.-R.; Gornitz, E. Physica E 2003, 17, 433.

(4) Rogach, A. L.; Kotov, N. A.; Koktysh, D. S.; Ostrander, J. W.; Ragoisha, G. A. Chem. Mater. 2000, 12, 2721.

(5) Meseguer, F.; Blanco, A.; Migues, H.; Garcia-Santamaria, F.; Ibisate, M.; Lopes, C. Colloids Surf. A 2002, 202, 281.

(6) Kubo, S.; Gu, Z.; Takahashi, K.; Ohko, Y.; Sato, O.; Fujishima, A. J. Am. Chem. Soc. 2002, 124, 10950.

(7) Joannopoulos, J. D.; Meade, R. D.; Winn, J. N. Photonic Crystals: Molding the Flow of Light; Princeton University Press: New York, 1995.

(8) (a) Asher, S. A. Crystalline Colloidal Narrow Band Radiation Filter, U.S. Patent No. 4,627,689, December 9, 1986. (b) Asher, S. A. Crystalline Colloidal Narrow Band Radiation Filter, U.S. Patent No. 4,632,517, December 30, 1986. (c) Carlson, R. J.; Asher, S. A. Appl. Spectrosc. 1984 38, 3. (d) Asher, S. A.; Flaugh, P. L.; Washinger, G. Spectroscopy (Eugene, Oreg.) 1986, 1, 26.

(9) (a) Asher, S. A.; Holtz, J.; Liu, L.; Wu, Z. J. Am. Chem. Soc. 1994 116, 4997. (b) Asher, S. A.; Jagannathan, S. Method of Making Solid Crystalline Narrow Band Radiation Filter, U.S. Patent No. 5,281,370, January 25, 1994. (c) Haacke, G.; Panzer, H. P.; Magliocco, L. G.; Asher, S. A. Narrow Band Radiation Filter Films, U.S. Patent No. 5,266,238, November 30, 1993.
(10) (a) Li, Y.; Tanaka, T. Annu. Rev. Mater. Sci. 1992, 22, 243. (b) Osada, Y.; Gong, J. Prog. Polym. Sci. 1993, 18, 187.

(11) (a) Irie, M. Adv. Polym. Sci. 1993, 110, 49. (b) Harmon, M. E.; Kuckling, D.; Frank, C. W. Macromolecules 2003, 36, 162. (c) Kang, M.S.; Gupta, V. K. J. Phys. Chem. B 2002, 106, 4127.

(12) Holtz, J. H.; Asher, S. A. Nature 1997, 389, 829.

(13) Weissman, J. M.; Sunkara, H. B.; Tse, A. S.; Asher, S. A. Science 1996, 274, 959.

(14) Lee, K.; Asher, S. A. J. Am. Chem. Soc. 2000, 122, 9534.

(15) (a) Kamenjicki, M.; Lednev, I.; Mikhonin, A.; Kasavamoorthy, R.; Asher, S. A. Adv. Funct. Mater. 2003, 13, 774. (b) Kamenjicki, M.; Lednev, I.; Asher, S. A. submitted to Adv. Funct. Mater., 2004.

(16) Paul, S.; Ranby, B. J. Polym. Sci., Polym. Chem. Ed. 1976, 14 2449.

(17) Hild, G.; Lamps, J. P.; Rempp, P. Polymer 1993, 34, 2875

(18) Iwakura, Y.; Kurosaki, T.; Ariga, N.; Ito, T. Makromol. Chem. 1966 97, 128

(19) Fitch, R. M. Polymer Colloids: A Comprehensive Introduction; Academic Press: San Diego, CA, 1997.

(20) Hermanson, G. T. Bioconjugate Techniques; Academic Press: San Diego, CA, 1996

(21) Pan, G.; Tse, A. S.; Kasavamoorthy, R.; Asher, S. A. J. Am. Chem. Soc. 1998, 120, 6518.

(22) Maack, J.; Ahuja, R. C.; Tachibana, H. J. Phys. Chem. 1995, 99 , 9210.

(23) Lednev, I. K.; Ye, Y.-Q.; Hester, R. E.; Moore, J. N. J. Phys. Chem. 1996, 100,13338

(24) Lednev, I. K.; Ye, Y.-Q.; Matousek, P.; Towrie, M.; Foggi, P.; Neuwahl, F. V. R.; Umapathy, S.; Hester, R. E.; Moore, J. N. Chem. Phys. Lett. 1998, 290, 68 .

(25) Rau, H. Angew. Chem., Int. Ed. 1973, 12, 224.

(26) Griffiths, J. Colour and Constitution of Organic Molecules; Academic Press: London, 1976.

(27) Crano, J. C., Guglielmetti, R. J., Eds. Organic Photochromic and Thermochromic Compounds; Plenum Press: New York, 1999. 